\title{
A Perfectly Matched Layer for the Acoustic Conservation Equations in the Time Domain
}

\author{
Andreas Hüppe \\ Manfred Kaltenbacher \\ Chair of Applied Mechatronics, \\ Alps Adriatic University, \\ Klagenfurt, Austria \\ andreas.hueppe@uni-klu.ac.at \\ http://am.uni-klu.ac.at \\ Received (Day Month Year) \\ Revised (Day Month Year)
}

\begin{abstract}
In recent years the development of free field radiation conditions in the time domain has become a topic of intensive research. Perfectly matched layer (PML) approaches for the frequency domain are well known. In the time domain, on the other hand, they suffer in many cases from highly increased complexity or instabilities. In this paper, we introduce a PML for the conservation equations of linear acoustics. The used formulation requires three auxiliary variables in $3 \mathrm{D}$ setups and circumvents thereby convolution integrals and higher order time derivatives. Furthermore we show the weak stability of the proposed formulation and show their good absorption properties by the means of numerical examples.
\end{abstract}

Keywords: Perfectly Matched Layer; time Domain; acoustics.

\section{Introduction}

Free field radiation boundary conditions are essential for every computational scheme for acoustic fields. In recent years a big variety of methods were proposed to avoid reflections on the outer boundary of the simulation domain. First order absorbing boundary conditions are one of the simpler methods and are only capable to absorb waves impinging normal to the boundary ${ }^{1}$. Higher order absorbing boundary conditions can overcome this limitation and show good results for any angle of incidence ${ }^{2,3}$ but require a special treatment of corner nodes. Another way is to use perfectly matched layers (PML). The idea is to surround the computational domain with an absorbing layer of elements in which the wave is damped without any reflections on the interface between propagation region and PML region. Hance the term perfectly matched. Initially proposed by Bérenger ${ }^{4}$ for electromagnetic wave propagation, the technique can be applied for frequency domain calculations in acoustics ${ }^{5-8}$. When it comes to time domain formulations the straight forward application of the PML technique leads to convolution integrals which would require complex and computationally expensive schemes. This drawback can be avoided by introducing higher order time derivatives $^{9}$ or using additional auxiliary variables inside the PML region ${ }^{10-12}$. Recently a stable finite difference formulation for the acoustic wave equation has been presented ${ }^{12}$ in which 
four additional unknowns are used for the three-dimensional case. F.Q. Hu ${ }^{11}$ also used auxiliary variables to obtain an unsplit PML formulation for the linearized Euler equations in context of the finite difference method. One additional variable for each unknown is used in this formulation.

A time domain PML for the conservation equations of linear acoustics is proposed in this contribution. With three additional unknowns in 3D, higher order time derivatives and convolution integrals can be avoided. The PML is incorporated in a spectral element calculation scheme proposed by G. Cohen ${ }^{13}$ which allows efficient calculations in the time domain with a high accuracy ${ }^{14}$.

The paper in hand is organized as follows. In section 2 we give the finite element formulation of the acoustic conservation equations. The PML in frequency and time domain is then derived in section 3. For the time dependent formulation we investigate the stability according to ${ }^{15,16}$ in section 3.3 and show the accuracy and absorption properties by the means of numerical examples in section 4 .

\section{Finite Element Formulation for the Conservation Equations}

The application of the finite element method to the second order wave equation for acoustics is well known and established. This scalar PDE can be derived from a system of partial differential equations known as the conservation equations of linear acoustics

$$
\begin{array}{r}
\frac{1}{\rho_{0} c^{2}} \frac{\partial p^{\prime}}{\partial t}+\nabla \cdot \vec{v}^{\prime}=F \\
\rho_{0} \frac{\partial \vec{v}^{\prime}}{\partial t}+\nabla p^{\prime}=0,
\end{array}
$$

where $p^{\prime}$ denotes the acoustic pressure, $\vec{v}^{\prime}$ the acoustic particle velocity and $F$ some source term. Furthermore, $\rho_{0}$ and $c$ are the density and speed of sound in the medium of propagation. Equations (1) and (2) can be seen as a subset of the Euler equations for isotropic media with no flow.

The first step in the finite element approximation is the derivation of the variational form. Equation (1) is multiplied with a testfunction $\varphi$ and (2) with a function $\vec{\psi}$. Integration over the computational domain $\Omega$ with boundary $\Gamma$ leads to the saddle point problem

$$
\begin{gathered}
\frac{1}{\rho_{0} c^{2}} \frac{\partial}{\partial t} \int_{\Omega} p^{\prime} \varphi \mathbf{d} \Omega+\int_{\Omega} \nabla \varphi \cdot \vec{v}^{\prime} \mathbf{d} \Omega-\int_{\Gamma} \varphi \vec{v} \cdot \vec{n} \mathbf{d} \Gamma=\int_{\Omega} \varphi F \mathbf{d} \Omega \\
\rho_{0} \frac{\partial}{\partial t} \int_{\Omega} \vec{v}^{\prime} \cdot \vec{\psi} \mathbf{d} \Omega+\int_{\Omega} \nabla p^{\prime} \cdot \vec{\psi} \mathbf{d} \Omega=0 .
\end{gathered}
$$

In order to achieve a stable finite element approximation, the two unknowns, particle velocity and pressure, have to be defined in different Sobolev spaces ${ }^{17,18}$. Let $L_{2}$ be the space of Lebesque integrable functions, $H^{1}$ the space of functions who's first derivatives also are in $L_{2}$ and $H$ (div) the space of functions who's divergence is in $L_{2}$. There are two stable combinations available which fulfill the Ladyzhenskaya-Babuška-Brezzi (or inf-sup) condition ${ }^{19}$. The two possibilities are: 
(1) Choose $p^{\prime} \in H(\operatorname{div})$ and $\vec{v}^{\prime} \in H^{1}$

(2) Choose $p^{\prime} \in H^{1}$ and $\vec{v}^{\prime} \in L_{2}$

The first choice could be realized by using Raviart-Thomas elements ${ }^{20}$. A better suited choice is presented by G. Cohen ${ }^{13}$ for nodal Lagrange elements. This method has advantages in time domain computaitons which is discussed briefly in the following.

Assume a domain, discretized by $N$ quadrilateral or hexahedral finite elements $K, \Omega=$ $\cup_{j=1}^{N} K_{j}$. Furthermore, there is a bijective mapping $\mathbf{F}_{\mathbf{j}}$ to transform the grid element $K_{j}$ to the reference element $\hat{K}_{j}$. Then the discrete spaces of approximation are defined as

$$
\begin{aligned}
& p_{h}^{\prime}, \varphi_{h} \in U_{h}^{k}=\left\{q \in H_{0}^{1}|q|_{K_{j}} \circ F_{j} \in Q^{k}\left(\hat{K}_{j}\right) \text { and } q=0 \text { on } \Gamma\right\} \\
& v_{h}^{\prime}, \psi_{h} \in V_{h}^{k}=\left\{w \in\left[L_{2}\right]^{d}\left|\frac{1}{\left|\mathcal{J}_{j}\right|} \mathcal{J}_{j} w\right|_{K_{j}} \circ F_{j} \in\left[Q^{k}\left(\hat{K}_{j}\right)\right]^{d}\right\} .
\end{aligned}
$$

Where $\mathcal{J}_{j}$ is the Jacobian of element $K_{j}$ and $Q^{k}\left(\hat{K}_{j}\right)$ is the set of Lagrange polynomials of degree $k$ and dimension $d$ on $\hat{K}_{j}$.

It is important to notice, that the space $V_{h}^{k}$ is define with the $H($ div)-conforming Piola transform ${ }^{20}$ form the grid element to the reference element, i.e. $v_{h}=1 / \mathcal{J}_{j} \mathcal{J}_{j} \hat{v}_{h}$. Because of this, the identity

$$
\int_{K_{j}} \nabla \varphi_{h} \cdot \vec{v}_{h}^{\prime} \mathbf{d} K_{j}=\int_{\hat{K}_{j}} \hat{\nabla} \hat{\varphi}_{h} \cdot \hat{v}_{h}^{\prime} \mathbf{d} \hat{K}_{j}
$$

holds for the stiffness integral in (3) and similarly for the corresponding integral in (4). Thereby, the element matrix is independent of the geometry and the global stiffness matrix is sparse and only depends on the connectivity of the elements in the grid.

Another important consequence is related to the choice of the discontinuous space $L_{2}$. Whereas the pressure unknowns are approximated continuously, the velocity unknowns are defined locally on each element. The practical effect of this fact is depicted in Fig. 1.

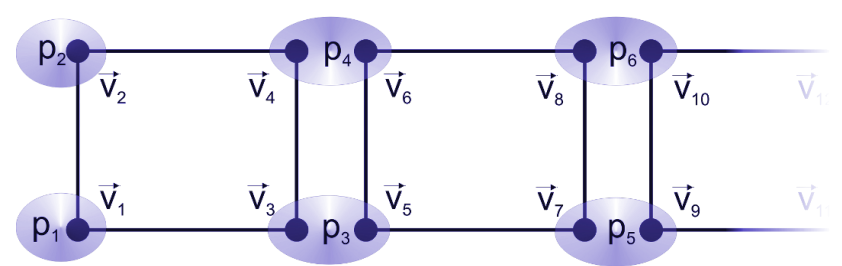

Fig. 1: Definition of unknowns due to mixed approximation

In a last step, spectral finite elements (s-FEM) ${ }^{21}$ of arbitrary order are used to discretize the domain. In this approach Lagrange polynomials are used in combination with a GaussLobatto integration rule. For quadrilateral and hexahedral elements the extension of the Shape functions can be obtained by a tensor product of the one dimensional functions. 


\section{A. Hüppe, M. Kaltenbacher}

Triangular and tetrahedral spectral elements require a more sophisticated construction and are still a matter of research ${ }^{22,23}$.

The finite element discretization leads to the matrix system

$$
\left(\begin{array}{cc}
\mathbf{D} & \mathbf{0} \\
\mathbf{0} & \mathbf{B}
\end{array}\right)\left(\begin{array}{c}
\dot{\mathbf{p}}^{\prime} \\
\dot{\mathbf{v}}^{\prime}
\end{array}\right)+\left(\begin{array}{cc}
\mathbf{0} & -\mathbf{R} \\
\mathbf{R}^{T} & \mathbf{0}
\end{array}\right)\left(\begin{array}{l}
\mathbf{p}^{\prime} \\
\mathbf{v}^{\prime}
\end{array}\right)=\left(\begin{array}{l}
\mathbf{F} \\
\mathbf{0}
\end{array}\right)
$$

Where the stiffness matrix is sparse and the mass matrix is (block-)diagonal due to the spectral element approximation.

It is obvious that a finite element solution of the conservation equations requires much more computational effort due to the fact that one has to compute also the discontinuous velocity unknowns in the domain. Nevertheless, this drawback can be overcome by utilizing the properties of the matrices and using explicit time stepping schemes thus giving an efficient time domain method which can be an alternative to computations on the second order wave equation ${ }^{14}$.

In order to make the scheme usable for a wider range of applications free field radiation boundary conditions are of great importance. A first order absorbing boundary condition for the time domain can be incorporated easily into the scheme. The boundary integral in (3) can be rewritten using the relation $\vec{v}^{\prime} \cdot \vec{n}=\left(1 / Z_{a}\right) p^{\prime}$, where $Z_{a}$ is the acoustic impedance of the medium ${ }^{24}$. By using the characteristic impedance of the medium, $Z_{a}=Z_{0}=\rho_{0} c$, we obtain the equality

$$
\int_{\Gamma} \varphi \vec{v}^{\prime} \cdot \vec{n} \mathbf{d} \Gamma=\int_{\Gamma} \frac{1}{Z_{0}} \varphi p^{\prime} \mathbf{d} \Gamma
$$

It should be noted, that this approximation is only exact for plane waves impinging orthogonal on the boundary and a more sophisticated approach is needed to achieve absorption at any angle of incidence of more complex waveforms.

\section{Perfectly Matched Layer}

Perfectly matched layers have been proven to show excellent absorption properties for waves at any angle of incidence ${ }^{4}$. Here, we follow Chew et al. ${ }^{7}$ and introduce a complex coordinate stretching. The space derivative in $\mathrm{x}$-direction is thereby written as

$$
\frac{\partial}{\partial x} \rightarrow \frac{j \omega}{j \omega+\sigma_{x}} \frac{\partial}{\partial x}=\zeta_{x} \frac{\partial}{\partial x}
$$

Analogously, this is done for the other two space dimensions $y$ and $z$. In the frequency domain, this change of variables can be directly applied to (1) and (2) thus obtaining the PML formulation. 


\subsection{Frequency Domain Formulation}

First we investigate the time dependent integral of (3). To evaluate the integral on the reference element $\hat{K}_{j}$ we apply a transformation using the Jacobian determinant and get

$$
\frac{\partial}{\partial t} \int_{K_{j}} \frac{1}{\rho_{0} c^{2}} p^{\prime} \varphi \mathbf{d} K_{j}=\frac{\partial}{\partial t} \int_{\hat{K}_{j}} \frac{1}{\rho_{0} c^{2}}\left|\mathcal{J}_{j}\right| \hat{p}^{\prime} \varphi \mathbf{d} \hat{K}_{j}
$$

The Jacobian determinant implies the computation of global derivatives which are transformed according to (10). By applying a Fourier transform we get the mass integral for the three dimensional case

$$
j \omega \int_{\hat{K}_{j}} \frac{1}{\rho_{0} c^{2}} \zeta_{x} \zeta_{y} \zeta_{z}\left|\mathcal{J}_{j}\right| \hat{p}^{\prime} \varphi \mathbf{d} \hat{K}_{j}
$$

Analogously, we have to apply this to the time dependent integral of (4). Special attention has to be paid definition of the functional space for the velocity unknowns. When mapping the integral onto the reference element we obtain

$$
\frac{\partial}{\partial t} \int_{K_{j}} \rho_{0} \vec{\psi} \cdot \vec{v}^{\prime} \mathbf{d} K_{j}=\rho_{0} \frac{\partial}{\partial t} \int_{\hat{K}_{j}} \frac{1}{\left|\mathcal{J}_{j}\right|} \mathcal{J}_{j} \mathcal{J}_{j}^{T} \hat{\vec{\psi}} \cdot \hat{\vec{v}}^{\prime} \mathbf{d} \hat{K}_{j}
$$

By using again the coordinate stretching and the Fourier transform we arrive at

$$
j \omega \int_{\hat{K}_{j}} \rho_{0} \frac{1}{\zeta_{x} \zeta_{y} \zeta_{z}\left|\operatorname{det} \mathcal{J}_{j}\right|}[\zeta] \mathcal{J}_{j}\left([\zeta] \mathcal{J}_{j}\right)^{T} \hat{\vec{\psi}} \cdot \hat{\vec{v}}^{\prime} \mathbf{d} \hat{K}_{j}
$$

with

$$
[\zeta]=\left(\begin{array}{ccc}
\zeta_{x} & 0 & 0 \\
0 & \zeta_{y} & 0 \\
0 & 0 & \zeta_{z}
\end{array}\right)
$$

The stiffness integrals do not need any special treatment because they are independent of the element geometry. Also the mass matrices remain (block-) diagonal due to the usage of the s-FEM. Thereby, the sparsity pattern of the matrices is not altered.

As stated above, the main advantage of the method is in time domain computations. When trying to transform the frequency formulation directly back into the time domain we observe convolution integrals or higher order time derivatives both of which would increase the complexity of the scheme significantly. In the next section we derive a time domain PML without these drawbacks.

\subsection{Time Domain Formulation}

In a first step we investigate (2) in its homogeneous frequency domain formulation. The introduction of stretched coordinates for each space direction reads as

$$
j \omega \rho_{0} \vec{v}^{\prime}+[\zeta] \nabla p^{\prime}=0 .
$$


A multiplication with $[\zeta]^{-1}$ then yields a PDE which can easily be transformed back into the time domain. By considering the definition of the damping matrix $[\zeta]$ we obtain

$$
j \omega \rho_{0} \vec{v}^{\prime}+\rho_{0}[\sigma] \vec{v}^{\prime}+\nabla p=0,
$$

in which we use the matrix of real valued damping factors

$$
[\sigma]=\left(\begin{array}{ccc}
\sigma_{x} & 0 & 0 \\
0 & \sigma_{y} & 0 \\
0 & 0 & \sigma_{z}
\end{array}\right) .
$$

In a second step, we consider (1) in its frequency domain formulation with the stretched coordinate transformation (10)

$$
j \omega \frac{1}{\rho_{0} c^{2}} p^{\prime}+\zeta_{x} \frac{\partial v_{x}^{\prime}}{\partial x}+\zeta_{y} \frac{\partial v_{y}^{\prime}}{\partial y}+\zeta_{z} \frac{\partial v_{z}^{\prime}}{\partial z}=0 .
$$

Now we add to (19) the neutral term

$$
\pm \frac{\sigma_{x}}{j \omega+\sigma_{x}} \frac{\partial v_{x}^{\prime}}{\partial x} \pm \frac{\sigma_{y}}{j \omega+\sigma_{y}} \frac{\partial v_{y}^{\prime}}{\partial y} \pm \frac{\sigma_{z}}{j \omega+\sigma_{z}} \frac{\partial v_{z}^{\prime}}{\partial z},
$$

and obtain the relation

$$
\begin{aligned}
j \omega \frac{1}{\rho_{0} c^{2}} p^{\prime} & +\left(\frac{\partial v_{x}^{\prime}}{\partial x}-\frac{\sigma_{x}}{j \omega+\sigma_{x}} \frac{\partial v_{x}^{\prime}}{\partial x}\right) \\
& +\left(\frac{\partial v_{y}^{\prime}}{\partial y}-\frac{\sigma_{y}}{j \omega+\sigma_{y}} \frac{\partial v_{y}^{\prime}}{\partial y}\right) \\
& +\left(\frac{\partial v_{z}^{\prime}}{\partial z}-\frac{\sigma_{z}}{j \omega+\sigma_{z}} \frac{\partial v_{z}^{\prime}}{\partial z}\right)=0 .
\end{aligned}
$$

The transformation of (21) back to the time domain is still not possible without convolution integrals or higher order time derivatives. To obtain a better suited form, we introduce an auxiliary variable for each space direction

$$
q_{x}=\frac{1}{j \omega+\sigma_{x}} \frac{\partial v_{x}^{\prime}}{\partial x} ; \quad q_{y}=\frac{1}{j \omega+\sigma_{y}} \frac{\partial v_{y}^{\prime}}{\partial y} ; \quad q_{z}=\frac{1}{j \omega+\sigma_{z}} \frac{\partial v_{z}^{\prime}}{\partial z} .
$$

By incorporating $\vec{q}=\left(q_{x}, q_{y}, q_{z}\right)^{T}$ into (21) we get the following system

$$
\begin{aligned}
j \omega \frac{1}{\rho_{0} c^{2}} p^{\prime}+\nabla \cdot \vec{v}^{\prime}+\left(\sigma_{x}, \sigma_{y}, \sigma_{z}\right)^{T} \cdot \vec{q} & =0, \\
q_{x}-\frac{1}{j \omega+\sigma_{x}} \frac{\partial v_{x}^{\prime}}{\partial x} & =0, \\
q_{y}-\frac{1}{j \omega+\sigma_{y}} \frac{\partial v_{y}^{\prime}}{\partial y} & =0, \\
q_{z}-\frac{1}{j \omega+\sigma_{z}} \frac{\partial v_{z}^{\prime}}{\partial z} & =0 .
\end{aligned}
$$


Now it is possible to transform each component into the time domain which yields the final system of partial differential equations

$$
\begin{aligned}
\frac{1}{\rho_{0} c^{2}} \frac{\partial p^{\prime}}{\partial t}+\nabla \cdot \vec{v}^{\prime}+\left(\sigma_{x}, \sigma_{y}, \sigma_{z}\right)^{T} \cdot \vec{q} & =0 \\
\rho_{0} \frac{\partial \vec{v}^{\prime}}{\partial t}+\rho_{0}[\sigma] \vec{v}^{\prime}+\nabla p^{\prime} & =0 \\
\frac{\partial \vec{q}}{\partial t}+[\sigma] \vec{q}-\mathcal{B} \vec{v}^{\prime} & =0 .
\end{aligned}
$$

The differential operator $\mathcal{B}$ is defined as

$$
\mathcal{B}=\left(\begin{array}{ccc}
\frac{\partial}{\partial x} & 0 & 0 \\
0 & \frac{\partial}{\partial y} & 0 \\
0 & 0 & \frac{\partial}{\partial z}
\end{array}\right)
$$

Transferring (24) to its weak formulation and using spectral elements then leads to the matrix system

$$
\left(\begin{array}{ccc}
\mathbf{D} & \mathbf{0} & \mathbf{0} \\
\mathbf{0} & \mathbf{B} & \mathbf{0} \\
\mathbf{0} & \mathbf{0} & \mathbf{M}
\end{array}\right)\left(\begin{array}{c}
\dot{\mathbf{p}}^{\prime} \\
\dot{\mathbf{v}}^{\prime} \\
\dot{\mathbf{q}}
\end{array}\right)+\left(\begin{array}{ccc}
\mathbf{0} & -\mathbf{R} & \mathbf{R}_{\mathbf{q}} \\
\mathbf{R}^{T} & \rho_{0} \mathbf{B}_{\sigma} & \mathbf{0} \\
\mathbf{0} & -\mathbf{R}_{\mathbf{v}} & \mathbf{M}_{\sigma}
\end{array}\right)\left(\begin{array}{l}
\mathbf{p}^{\prime} \\
\mathbf{v}^{\prime} \\
\mathbf{q}
\end{array}\right)=\left(\begin{array}{l}
\mathbf{0} \\
\mathbf{0} \\
\mathbf{0}
\end{array}\right)
$$

It can be seen immediately that the global stiffness matrix is no longer symmetric. But the mass matrix $\mathbf{M}$ on the other hand is a diagonal matrix due to the s-FEM discretization. Therefore, it is still possible to use explicit time discretization schemes in which the inconvenient structure of the stiffness matrix has only minor influence on the computational time. Furthermore, the auxiliary variable and the additional matrices have to be evaluated and stored only inside the PML domain.

\subsection{Stability}

For the usability, the stability of the proposed formulation is of great importance. To give not only numerical examples for the stability we follow the theory for hyperbolic systems as stated by D. Appelö et al. ${ }^{15}$.

For discussion, we consider the constant coefficient Cauchy problem

$$
\frac{\partial u(\vec{x}, t)}{\partial t}=P(\partial / \partial \vec{x}) u(\vec{x}, t), \quad u(\vec{x}, 0)=u_{0}(\vec{x}), \quad \vec{x} \in \mathbb{R}^{d}, \quad 0<t<T,
$$

with the constant coefficient operator $P(\partial / \partial \vec{x})$. To perform the analysis, a Fourier transform in space is applied to the operator $P$ which leads to a system of ordinary differential equations

$$
\frac{\partial u(\vec{k}, t)}{\partial t}=P(i \vec{k}) u(\vec{k}, t), \quad u(\vec{k}, 0)=u_{0}(\vec{k}), \quad \vec{k} \in \mathbb{R}^{s}, \quad 0<t<T .
$$

Weak and strong stability are then defined by ${ }^{15}$ : 
Definition 3.1. The Cauchy problem (27) is

(1) strongly stable if all solutions satisfy an estimate

$$
\|u(\cdot, t)\|_{L_{2}} \leq K\|u(\cdot)\|_{L_{2}} \cdot
$$

(2) weakly stable if all solutions satisfy an estimate

$$
\|u(\cdot, t)\|_{L_{2}} \leq K(1+t)^{s}\|u(\cdot)\|_{H^{s}},
$$

where $s>0$.

The condition

$$
\mathfrak{R e}\left\{\lambda_{j}(P(i \vec{k}))\right\} \leq 0,
$$

on the real part of all eigenvalues $\lambda_{j}$ of $P(i \vec{k})$ is necessary and sufficient to ensure weak stability of the formulation. If all eigenvalues have a strictly negative real part, strong stability can be shown. Even for the case of eigenvalues with real part equal to zero, strong stability can also be shown if the corresponding eigenvectors span their respective invariant subspace, i.e. there is a full set of eigenvectors.

\subsubsection{Two-dimensional case}

For a better readable analysis (24) is written in the form

$$
U_{t}=A U_{x}+B U_{y}+D,
$$

where

$$
U=\left(p, v_{x}, v_{y}, q_{x}, q_{y}\right)^{T},
$$

and the coefficient matrices are defined as

$$
\begin{gathered}
A=\left(\begin{array}{ccccc}
0 & -c^{2} \rho_{0} & 0 & 0 & 0 \\
-1 / \rho_{0} & 0 & 0 & 0 & 0 \\
0 & 0 & 0 & 0 & 0 \\
0 & 1 & 0 & 0 & 0 \\
0 & 0 & 0 & 0 & 0
\end{array}\right), \quad B=\left(\begin{array}{ccccc}
0 & 0 & -c^{2} \rho_{0} & 0 & 0 \\
0 & 0 & 0 & 0 & 0 \\
-1 / \rho_{0} & 0 & 0 & 0 & 0 \\
0 & 0 & 0 & 0 & 0 \\
0 & 0 & 1 & 0 & 0
\end{array}\right), \\
D=-\left(\begin{array}{ccccc}
0 & 0 & 0 & \sigma_{x} & \sigma_{y} \\
0 & \sigma_{x} & 0 & 0 & 0 \\
0 & 0 & \sigma_{y} & 0 & 0 \\
0 & 0 & 0 & \sigma_{x} & 0 \\
0 & 0 & 0 & 0 & \sigma_{y}
\end{array}\right) .
\end{gathered}
$$


According to ${ }^{16}$ we calculate the eigenvalues and eigenvectors of the principal part of $P(i k)$ and observe that the eigenvalues are

$$
\lambda_{1,2,3}(P(i k))=0 \quad, \quad \lambda_{4,5}(P(i k))= \pm i c \sqrt{k_{x}+k_{y}} .
$$

Therefore the system is weakly stable. Still, the zero eigenvalue has multiplicity three. As the set of eigenvectors is also incomplete, we cannot state strong stability.

\subsubsection{Three-dimensional case}

As done above, the system is written in the form

$$
U_{t}=A U_{x}+B U_{y}+C U_{z}+D .
$$

For the three dimensional case, the matrices are defined as

$$
\begin{gathered}
A=\left(\begin{array}{cccccccc}
0 & -c^{2} \rho_{0} & 0 & 0 & 0 & 0 & 0 \\
-1 / \rho_{0} & 0 & 0 & 0 & 0 & 0 & 0 \\
0 & 0 & 0 & 0 & 0 & 0 & 0 \\
0 & 0 & 0 & 0 & 0 & 0 & 0 \\
0 & 1 & 0 & 0 & 0 & 0 & 0 \\
0 & 0 & 0 & 0 & 0 & 0 & 0 \\
0 & 0 & 0 & 0 & 0 & 0 & 0
\end{array}\right), \quad B=\left(\begin{array}{cccccccc}
0 & 0 & -c^{2} \rho_{0} & 0 & 0 & 0 & 0 \\
0 & 0 & 0 & 0 & 0 & 0 & 0 \\
-1 / \rho_{0} & 0 & 0 & 0 & 0 & 0 & 0 \\
0 & 0 & 0 & 0 & 0 & 0 & 0 \\
0 & 0 & 0 & 0 & 0 & 0 & 0 \\
0 & 0 & 1 & 0 & 0 & 0 & 0 \\
0 & 0 & 0 & 0 & 0 & 0 & 0
\end{array}\right) \\
C=\left(\begin{array}{ccccccc}
0 & 0 & 0 & -c^{2} \rho_{0} & 0 & 0 & 0 \\
0 & 0 & 0 & 0 & 0 & 0 & 0 \\
0 & 0 & 0 & 0 & 0 & 0 & 0 \\
-1 / \rho_{0} & 0 & 0 & 0 & 0 & 0 & 0 \\
0 & 0 & 0 & 0 & 0 & 0 & 0 \\
0 & 0 & 0 & 0 & 0 & 0 & 0 \\
0 & 0 & 0 & 1 & 0 & 0 & 0
\end{array}\right), \quad D=-\left(\begin{array}{cccccccc}
0 & 0 & 0 & 0 & \sigma_{x} & \sigma_{y} & \sigma_{z} \\
0 & \sigma_{x} & 0 & 0 & 0 & 0 & 0 \\
0 & 0 & \sigma_{y} & 0 & 0 & 0 & 0 \\
0 & 0 & 0 & \sigma_{z} & 0 & 0 & 0 \\
0 & 0 & 0 & 0 & \sigma_{x} & 0 & 0 \\
0 & 0 & 0 & 0 & 0 & \sigma_{y} & 0 \\
0 & 0 & 0 & 0 & 0 & 0 & \sigma_{z}
\end{array}\right)
\end{gathered}
$$

The eigenvalues of the principle part are calculated as

$$
\lambda_{1, . ., 5}(P(i k))=0 \quad, \quad \lambda_{6,7}(P(i k))= \pm i c \sqrt{k_{x}+k_{y}+k_{z}} .
$$

Similar to the two dimensional case weak stability is thereby shown.

\section{Three dimensional numerical analysis}

In this section, the PML should be investigated in terms of accuracy and efficiency. As the properties of the proposed formulation are very similar between two and three space dimensions, all investigations will be done for a three dimensional setup discussed in the following. 


\subsection{Setup}

For a detailed analysis, the setup depicted in Fig. 2 (a) should be considered.

As an initial condition an acoustic pressure pulse at time $t=0$ is chosen with exponential distribution around the domains center at $r=\sqrt{x^{2}+y^{2}+z^{2}}=0$ given by

$$
\begin{aligned}
& p(t=0, r)=-\frac{1}{4 B} \mathbf{e}^{-B r^{2}}, \\
& \vec{v}(t=0, r)=\overrightarrow{0}
\end{aligned}
$$

For the actual simulation $B$ is set to 0.5 which leads to the initial acoustic pressure distripution shown on the left hand side of Fig. 2 (b) along the x-axis of the propagation region. For the numerical investigation, we discretize the propagation region of dimension

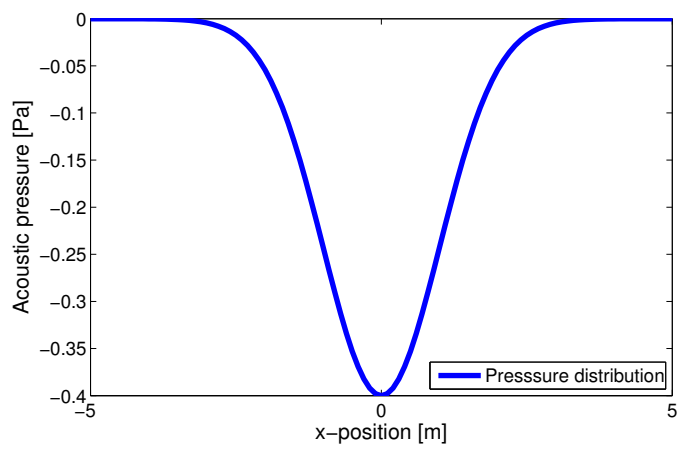

(a) Pressure distribution along thex-axis

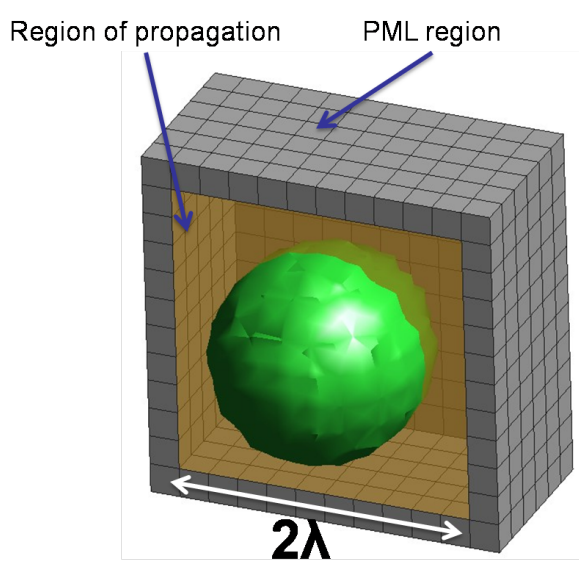

(b) Cut through PML and propagation regions including iso-surface of initial acoustic pressure

Fig. 2: Three dimensional setup.

$[-\lambda, \lambda]^{3}$ with $10^{3}$ elements, which corresponds to approximately 5 elements per wavelength. Due to this coarse discretization, linear finite elements are assumed to provide inaccurate results. As a consequence, computations with spectral elements of order two and three are performed as well.

As the mass matrix in (26) can be inverted analytically it is easily possible to apply explicit time stepping schemes. Here, a fourth order Runge-Kutta time stepping scheme is used for all simulations. The time step size is chosen to be one tenth of the limiting Courant-Friedrichs-Lewy (CFL) condition ${ }^{25}$ and the speed of sound is assumed to be $c=1$ $\mathrm{m} / \mathrm{s}$. 


\subsection{First numerical results}

In a first step, the performance of the proposed formulation should be tested qualitatively with the setup described above.

For the damping parameter $\sigma$ we choose a quadratic profile inside the PML as analysed in section 4.3. Due to the coarse discretization of only 5 elements per wavelength we choose quadratic spectral elements for the spacial discretization. Snapshots of the acoustic pulse
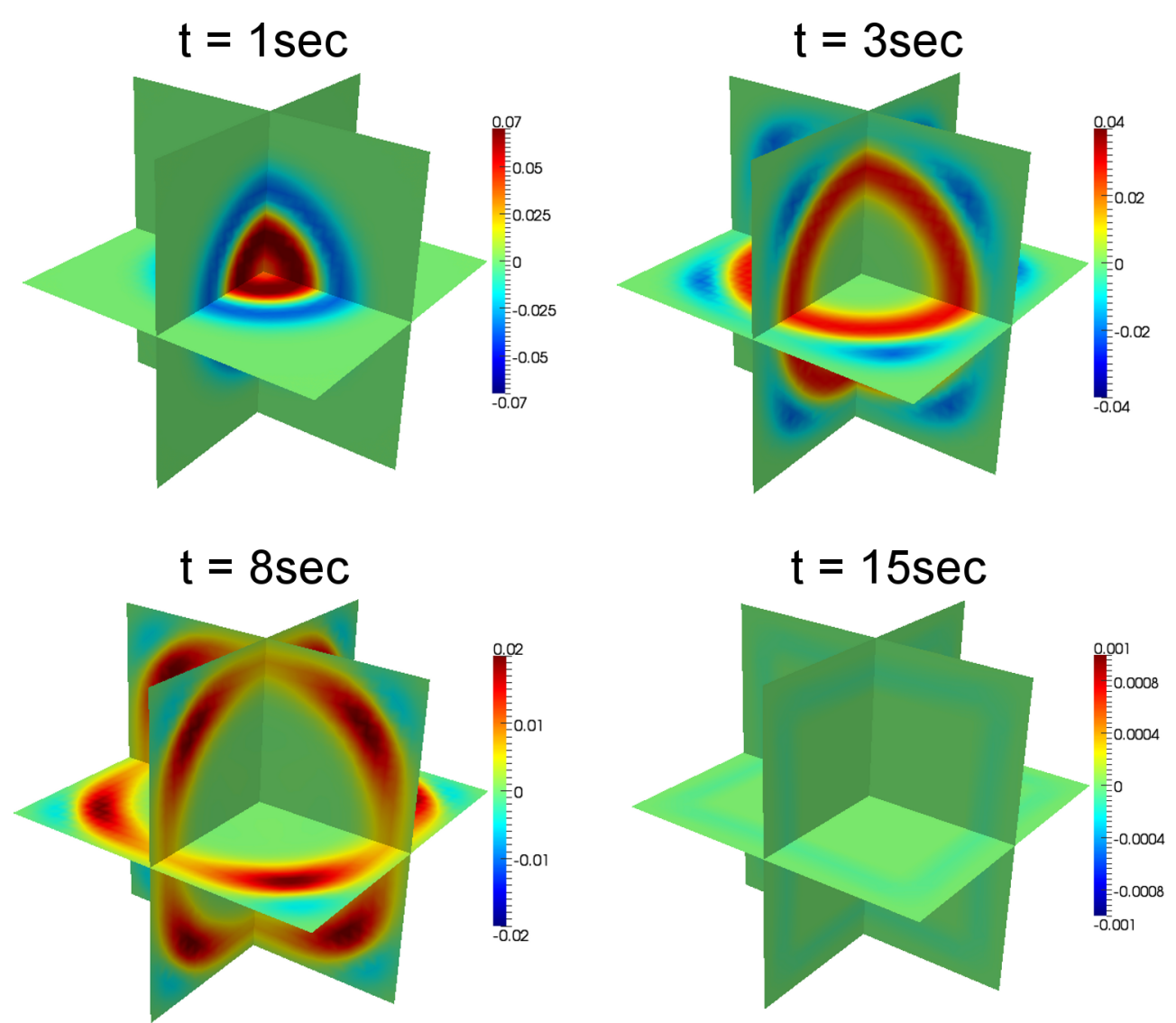

Fig. 3: Contour plots of acoustic pressure in the domain at different time levels.

propagation through the computational domain at different time levels are displayed in Fig. 3.

It is noticeable the wave enters the damping layer and is completely absorbed without any visible reflections at the end of the computation. For a better visibility, the color scale is adapted to meet the actual maximum and minimum amplitude of the pulse at each time.

To further investigate the properties of the scheme, a detailed analysis is performed in the following sections. 


\subsection{Choice of damping profile}

Still an open question is the optimal choice for the damping profile inside the PML. Lets assume a damping layer with length $L$. Four different schemes to calculate the damping coefficient at position $j$ inside the layer $(0 \leq j \leq L)$ should be compared.

\section{Constant damping}

In this case, the damping parameter is constant over the complete domain and calculated as $^{1}$

$$
\sigma_{0}=-\frac{c \ln R}{2 L},
$$

where $c$ is the speed of sound and $R$ the reflection coefficient.

\section{Quadratic damping}

When using a quadratic damping function, the parameter $\sigma_{i}$ starts with a value of zero at the interface between damping region and propagation region and is increased in $j$ direction by the functional

$$
\sigma_{j}^{q}=\sigma_{0}^{q} \frac{j^{2}}{L^{2}},
$$

where the constant factor $\sigma_{i}^{q}$ is chosen as

$$
\sigma_{0}^{q}=-\frac{3 c \ln R}{2 L} .
$$

\section{Inverse distance damping}

Here, the damping profile is increased towards the boundary by

$$
\sigma_{j}^{i}=\frac{c}{L-j} .
$$

Special attention has to be paid to this case in combination with the Gauss-Lobatto quadrature as used in the spectral element method. As there is an integration point at $j=L$ the damping factor becomes infinity here. To circumvent this problem, homogeneous boundary conditions on all variables are applied at the outer boundary of the PML.

\section{Alternative damping}

With the last function considered, the damping factor is zero at the interface and approaches a maximum value at the outer boundary. This profile is given s $^{12}$

$$
\sigma_{j}^{a}=\sigma_{0}^{a}\left(\frac{j}{L}-\frac{\sin \left(\frac{2 \pi j}{L}\right)}{2 \pi}\right) .
$$


The constant factor is calculated depending on the dimension of the layer by

$$
\sigma_{0}^{a}=\frac{c}{L} \log \left(\frac{1}{R}\right) q
$$

In all the simulation we choose $R=10^{-3}$ and calculate the acoustic energy inside the region of propagation according to

$$
w_{a}=\frac{1}{2}\left(\rho_{0} \vec{v}^{\prime} \cdot \vec{v}^{\prime}+\frac{1}{\rho_{0} c^{2}} p^{\prime 2}\right)
$$

The expected result is that the acoustic energy decays rapidly as the wave leaves the propagation region into the PML. From the theoretical point of view, the energy should approach its minimal value after approximately eleven seconds. The rate of energy decay can thereby serve as an indicator for the performance of the chosen damping function.

To perform the analysis, a PML thickness of $\lambda / 4$ is chosen which leads to three second order spectral elements in thickness direction. The results of the analysis are displayed
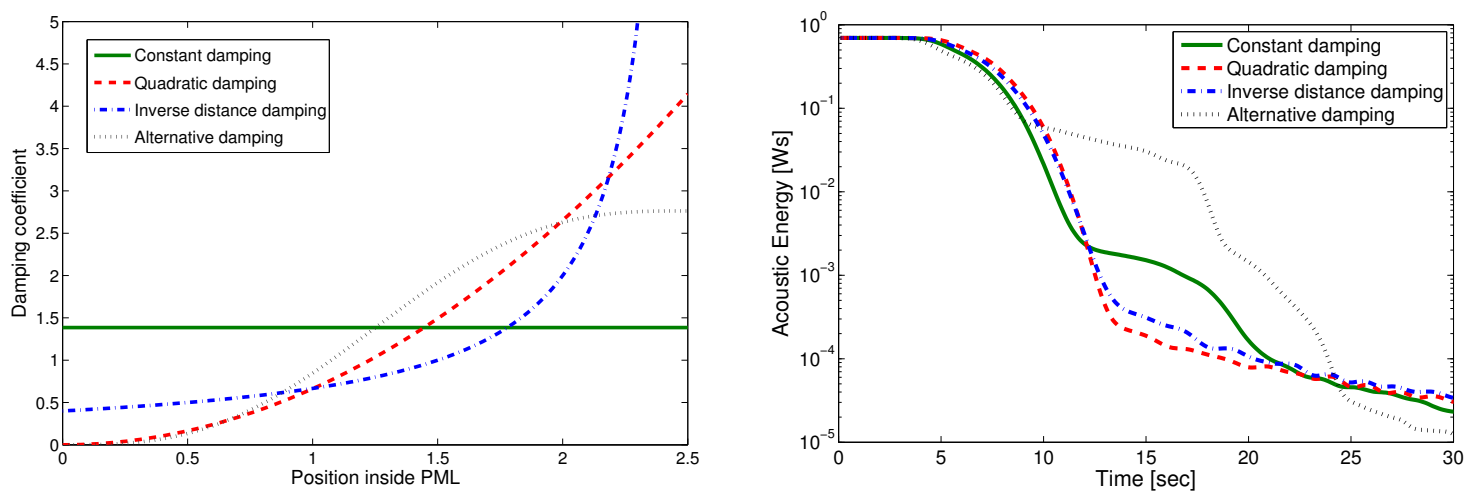

Fig. 4: Damping profiles (left) and acoustic energy decay in the propagation domain (right).

in Fig. 4 along with the damping profile for each considered method. It can be seen, that inverse distance and quadratic damping performs best when applied to the proposed scheme. Among these two, the latter shows a better performance in the range between 12 and 20 seconds. This is most likely due to the fact, that the inverse distance damping starts of with a jump of the damping coefficient on the interface between propagation and damping region which could cause reflections. 


\subsection{Numerical accuracy}

For the setup under investigation, the analytical solution can be given as ${ }^{26}$

$$
p(t, r)=-\frac{1}{4 B}\left(\frac{r+t}{2 r} \mathbf{e}^{-B(r+t)^{2}}-\frac{r-t}{2 r} \mathbf{e}^{-B(r-t)^{2}}\right) .
$$

Therefore it is possible to compute the $l_{2}$ error inside the region of propagation which gives an impression of the accuracy of the scheme and the influence of different PML configurations. This measure of error can be given as:

$$
E_{l_{2}}=\sqrt{\frac{1}{N} \sum_{i=1}^{N}\left(p_{h_{i}}^{\prime}-p_{i}^{\prime}\right)^{2}},
$$

where $N$ is the number of nodes inside the domain and $p_{i}^{\prime}$ denotes the analytical and $p_{i}^{\prime}$ the numerical solution at node $i$.

The following three setups are investigated along with different element orders:

(1) PML 1: Width of $\lambda / 4$ with 1 element inside the layer.

(2) PML 2: Width of $\lambda / 2$ with 3 elements inside the layer.

(3) PML 3: Width of $\lambda$ with 5 elements inside the layer.

The setup is chosen such that the wave enters the PML after 5 seconds. Pictured in Fig. 5 are the results of the analysis for different approximation orders. Its obvious that the accuracy of the computations is increased significantly when choosing higher order elements for approximation. Whereas, the error of the first order computation is in the range of $1 \%$ its reduced to $0.05 \%$ for the second order case and to $0.005 \%$ when using elements of order three. Starting from $t=5$ seconds, the effect of the different PML setups becomes visible. For all element types we observe an improvement of the absorption properties by choosing more elements inside the damping layer. For PML 1 with only one element the high error after the wave enters the PML indicates many reflections and bad absorption properties. In difference to this, it is visible that that the error is much smaller if there are more elements inside the PML. At worst the error is in the range of the approximation error which leads to the conclusion that the PML gives only minor reflections and does not decrease the accuracy of the computation.

\subsection{Stability}

The last investigation is subjected to the stability. Even though the analysis in section 3.3 has already shown weak stability of the formulation and no signs of instability occurred in the performed computations, it seems reasonable to test the scheme with longer simulation times and a constant sine excitation. For the pulse excitation, the acoustic energy inside the complete domain should decrease constantly over the simulation time. For the constant excitation, a stable scheme should provide a steady state oscillation of acoustic energy. As pictured in Fig. 6 both assumptions are verified for different orders of approximation. Even for much longer simulation times no instabilities occurred and the energy remains finite. 

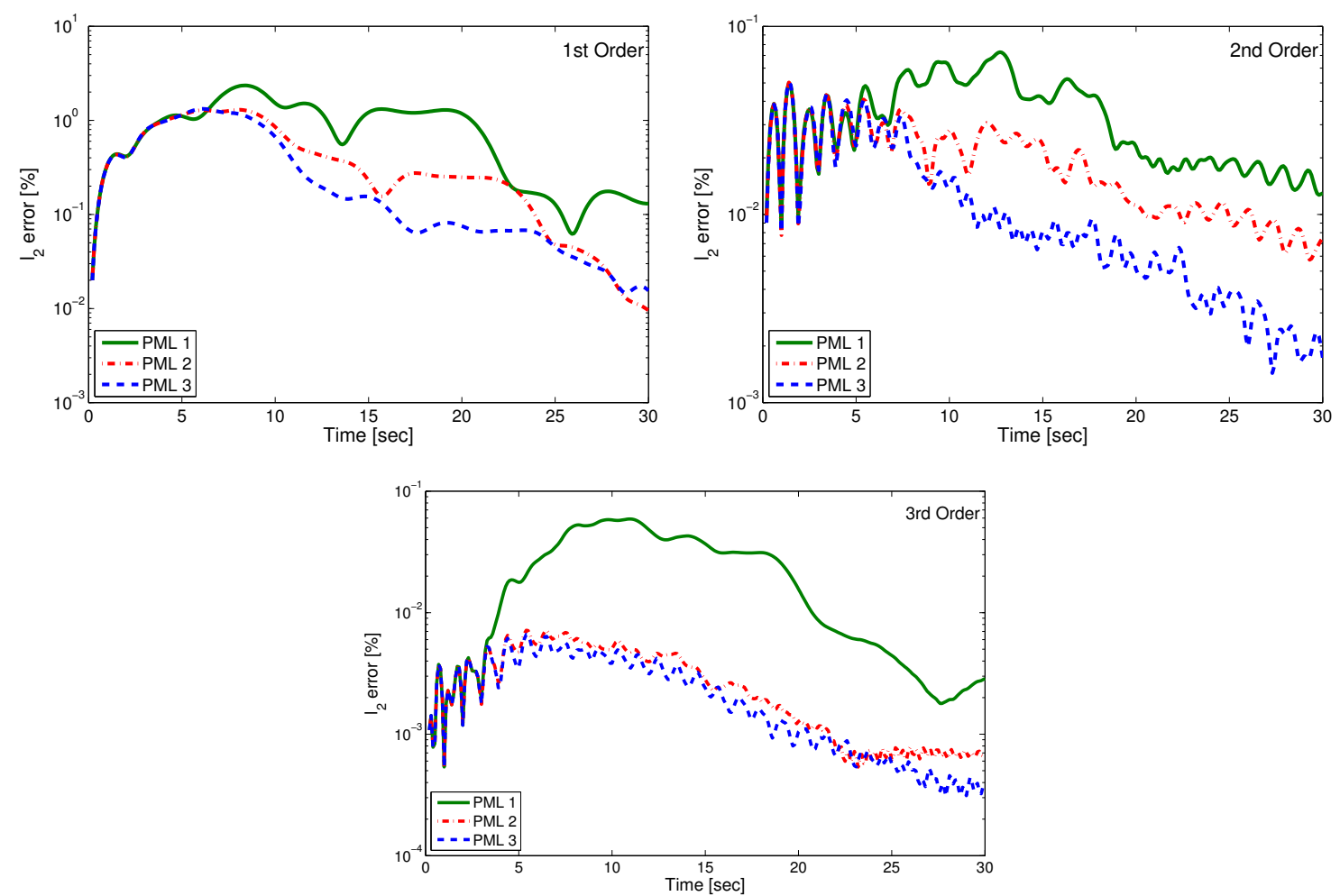

Fig. 5: Numerical error inside the propagation region for different PMLs and orders of approximation
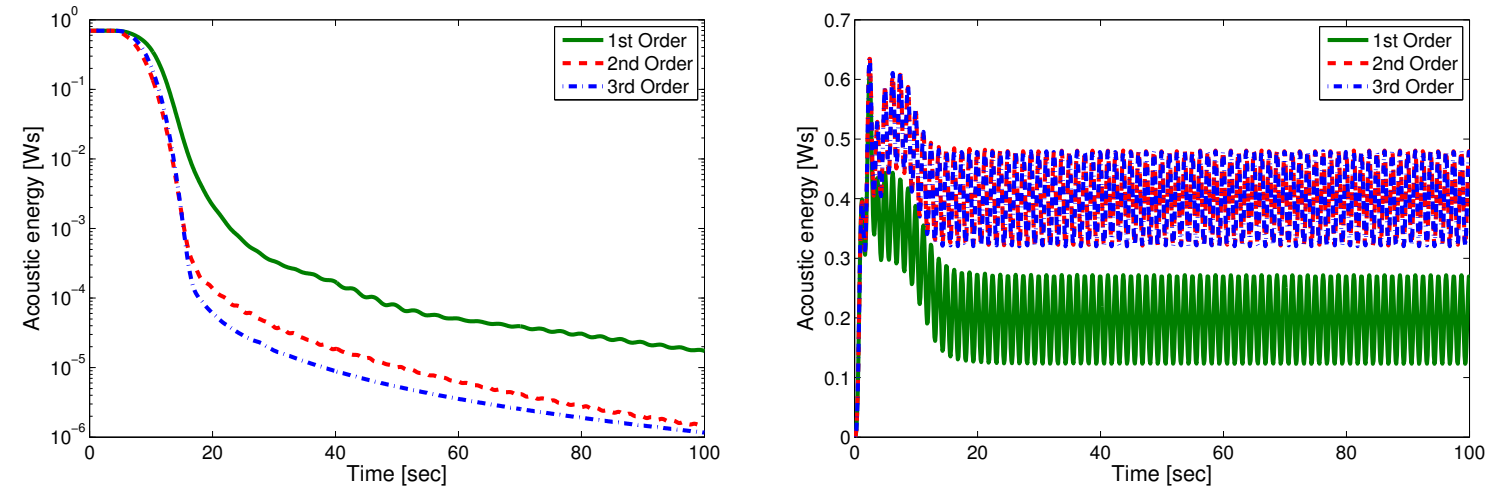

Fig. 6: Long term simulation of acoustic pulse (left) and constant sine excitation (right)

\section{Conclusion}

The basic computational scheme as introduced in section 2 has already shown its excellent approximation properties and performance. Now, the paper in hand proposes a novel per- 
fectly matched layer in the time domain for this scheme. The formulation requires only three auxiliary variables and does not require the evaluation of higher order time derivatives. After an mathematical investigation in which it was possible to show the stability of the scheme the formulation was tested against a numerical benchmark problem. It was possible to show that a quadratic damping profile is the best choice for the proposed method. Nevertheless, the quality of absorbtion with inverse distance damping was in the same range.

Numerical accuracy has been investigated in terms of acoustic energy and in comparison with an analytical solution. The results show that the performance of the PML also benefitts from a higher order approximation and that the maximum error wich is caused by reflections is in the range of the overal accuracy of the approximation. It was shown that one should use at least two layeres of absorbing elements are necessary to avoid reflections.

Finaly, the stability was investigated for a longer simulation time for pulse and constant sine excitation. No instabilities occured for the cases investigated.

The proposed PML shows excellent absorption properties, allows a straight forward implementation and improves the initial scheme to be applicable to freee field radiation problems in the time domain.

\section{References}

1. M. Kaltenbacher. Numerical Simulation of Mechatronic Sensors and Actuators. Springer, Berlin, 2. edition, 2007. ISBN: 978-3-540-71359-3.

2. Thomas Hagstrom, Assaf Mar-Or, and Dan Givoli. High-order local absorbing conditions for the wave equation: Extensions and improvements. Journal of Computational Physics, 227:3322$3357,2007$.

3. Eliane Bécache, Dan Givoli, and Thomas Hagstrom. High-order absorbing boundary conditions for anisotropic and convective wave equations. J. Comput. Phys., 229:1099-1129, February 2010.

4. Jean-Pierre Berenger. A perfectly matched layer for the absorption of electromagnetic waves. J. Comput. Phys., 114(2):185-200, 1994.

5. M. Kaltenbacher, M. Escobar, B. Flemisch, and B. Wohlmuth. Computational Aeroacoustics using Nonmatching Grids and PML. In Computational Electromagnetism and Acoustics, Oberwolfach Report 5, pages 45-48, 2007.

6. Alfredo Bermdez, Luis Hervella-Nieto, Andrs Prieto, and Rodolfo Rodrguez. An optimal finiteelement/pml method for the simulation of acoustic wave propagation phenomena. In Variational Formulations in Mechanics: Theory and Applications, 2006.

7. W. C. Chew, J. M. Jin, and E. Michielssen. Complex coordinate stretching as a generalized absorbing boundary condition. Microwave and Optical Technology Letters, 15:363-369, 1997.

8. Zhonghua $\mathrm{Wu}$ and Jiayuan Fang. High-performance pml algorithms. Microwave and Guided Wave Letters, IEEE, 6(9):335 -337, September 1996.

9. G. Cohen. Higher-Order Numerical Methods for Transient Wave Equations. Springer, 2002.

10. Fang Q. Hu. A stable, perfectly matched layer for linearized euler equations in unsplit physical variables. Journal of Computational Physics, 173(2):455-480, 2001.

11. Wenyi Hu, Aria Abubakar, and Tarek M. Habashy. Application of the nearly perfectly matched layer in acoustic wave modeling. Geophysics, 72(5):SM169-SM175, 2007.

12. Marcus J. Grote and Imbo Sim. Efficient pml for the wave equation. ArXiv e-prints, 2010.

13. G. Cohen and S. Fauqueux. Mixed finite elements with mass-lumping for the transient wave equation. Journal of Computational Acoustics, 8:171-188, 2000. 
14. Hüppe. Limits of finite element methods in the mid-frequency range. In Proceedings of the DAGA'09, pages 202-205, 2009. CD-ROM-Proceedings, Amsterdam.

15. Daniel Appelö, Thomas Hagstrom, and Gunilla Kreiss. Perfectly matched layers for hyperbolic systems: General formulation, well-posedness, and stability. SIAM Journal on Applied Mathematics, 67:1-23, 2006.

16. Heinz-Otto Kreiss and Jens Lorenz. Initial-Boundary Value Problems and the Navier-Stokes Equations. SIAM Classics in Applied Mathematics, 1989.

17. Michele Benzi, Gene H. Golub, and Jrg Liesen. Numerical solution of saddle point problems. ACTA NUMERICA, 14:1-137, 2005.

18. D. Breass. Finite Elemente. Springer, Berlin, 2002.

19. F. Brezzi and M. Fortin. Mixed and Hybrid Finite Element Methods. Springer, New York, 1991.

20. P. A. Raviart and J. M. Thomas. A mixed finite element method for 2nd order elliptic problems. Mathematical Aspects of the Finite Element Method, Lecture Notes in Mathematics, 606:292315, 1977.

21. C. Bernadi and Y. Maday. Spectral Methods. In J.L. Lions P.G. Ciarlet, editor, Handbook of Numerical Analysis, volume V: Techniques of Scientific Computing. Elsevier Science \& Technology, 1990.

22. Richard Pasquetti and Francesca Rapetti. Spectral element methods on unstructured meshes: Comparisons and recent advances. Journal of Scientific Computing, 27:377-387, 2006. 10.1007/s10915-005-9048-6.

23. F. Giraldo and M. Taylor. A diagonal-mass-matrix triangular-spectral-element method based on cubature points. Journal of Engineering Mathematics, 56:307-322, 2006. 10.1007/s10665-0069085-7.

24. D. T. Blackstock. Fundamentals of Physical Acoustics. Blackstock, 2000.

25. R. Courant, K. Friedrichs, and H. Lewy. On the partial difference equations of mathematical physics. IBM J. Res. Dev., 11:215-234, March 1967.

26. S. Zheng and M. Zhuang. Three-Dimensional Benchmark Problem for Broadband Time-Domain Impedance Boundary Conditions. AIAA Journal, 42:405-407, February 2004. 